\title{
Systemic Treatment Options in Hepatocellular Carcinoma
}

\author{
Lorenza Rimassa ${ }^{a} \quad$ Tiziana Pressiania $^{a} \quad$ Philippe Merle $^{b}$ \\ ${ }^{a}$ Medical Oncology and Hematology Unit, Humanitas Cancer Center, Humanitas Clinical \\ and Research Center - IRCCS, Milan, Italy; ${ }^{b}$ Hepatology Unit, Croix-Rousse Hospital, \\ Groupement Hospitalier Lyon Nord, Lyon, France
}

\section{Keywords}

Advanced hepatocellular carcinoma $\cdot$ Cabozantinib - Lenvatinib · Nivolumab ·

Pembrolizumab $\cdot$ Ramucirumab $\cdot$ Regorafenib · Sorafenib · Systemic therapy

\begin{abstract}
Background: Patients with advanced hepatocellular carcinoma (HCC) typically have poor survival outcomes. Until recently, sorafenib was the only systemic therapy option available and no agents were approved after sorafenib failure. However, rapid changes are beginning to emerge in the treatment landscape of advanced HCC, with approvals of regorafenib, nivolumab, lenvatinib, pembrolizumab, and cabozantinib and positive phase II/III clinical trial results with other agents. Summary: Here, we provide a comprehensive overview of the clinical trial data of systemic agents that are currently approved for advanced HCC (sorafenib, regorafenib, and nivolumab), including agents recently approved in 2018 (lenvatinib, pembrolizumab, and cabozantinib) and those with recent positive phase II/III results (ramucirumab). Key features of the clinical trial design, including patient selection criteria, the use of biomarkers in HCC, and criteria for efficacy assessment, and their implications in real-world practice are discussed. Important ongoing and planned trials in advanced HCC are summarized to provide a glimpse into the future of advanced HCC treatment. From a physician's viewpoint, the treatment algorithms for advanced HCC are undergoing significant changes, as additional and imminent approvals impact the choices of first- and second-line treatment and decisions regarding the timing of therapy initiation. With these additional choices at hand, treatment sequencing remains a complex task and should take patient selection and tolerance profiles
\end{abstract}


Rimassa et al.: Systemic Treatment Options in HCC

into account. Key Messages: The treatment of advanced HCC remains challenging and complex. The rapid developments in systemic therapy for advanced HCC should be considered when determining the best choice and sequence of treatment for patients with advanced HCC.

(c) 2019 S. Karger AG, Basel

\section{Introduction}

Primary liver cancer is among the leading causes of cancer-related death worldwide. Hepatocellular carcinoma (HCC) constitutes $80-90 \%$ of all primary liver cancers [1]. Major risk factors for HCC include chronic hepatitis B virus (HBV) and hepatitis C virus (HCV) infections, excessive alcohol consumption, nonalcoholic fatty liver disease, hemochromatosis, aflatoxin $\mathrm{B}_{1}$, and, more generally, all causes of cirrhosis [1].

The Barcelona Clinic Liver Cancer (BCLC) staging system is widely accepted for the evaluation of HCC stages and treatment allocation, as it is an effective, integrated system that takes into account the patient performance status (PS), tumor burden, vascular involvement, potential hepatic dysfunction, and portal hypertension [1]. The Hong Kong Liver Cancer (HKLC) staging system [2] may be more suitable for Asian patients than the BCLC system because of etiologic differences in HCC between Asian and European patients [3]. According to the Asian Pacific [4] and Japanese [5] guidelines, diagnostic imaging methods inform diagnosis, staging, and treatment strategies.

At early stages of HCC (BCLC stage 0/A), treatment strategies are potentially curative and include liver resection, liver transplantation, and ablation. At intermediate stages (BCLC stage B), palliative locoregional therapies such as transarterial chemoembolization (TACE) remain the gold standard for treatment [1]. However, most HCC cases are diagnosed at advanced stages (BCLC stage C) when curative therapies or TACE are no longer available [1].

The decision to move from locoregional treatment (TACE) to systemic therapy usually occurs at BCLC stage B or C. In patients with BCLC stage B (intermediate stage), it is recommended to stop TACE and initiate systemic therapy if the patient shows TACE toxicity, absence of a response, disease progression after 1 or 2 courses of TACE, or progression with vascular or extrahepatic spread (and thus has BCLC stage C) [1,6]. However, the observational OPTIMIS study showed that real-world TACE use deviates from guidelines in $39 \%$ of patients [7]. The HKLC system identifies subsets of BCLC intermediate- and advanced-stage patients who would benefit from more aggressive treatment [2], and patients with macrovascular invasion often receive TACE in Asian countries [3].

An earlier switch to systemic therapy is recommended to avoid toxicity, decompensated liver disease, and a general status deterioration due to prolonged TACE $[6,8]$ and because outcomes are improved $[9,10]$. However, the lack of prospectively evaluated criteria leads to heterogeneous decision-making in clinical practice. Prospective studies are needed to help define the time point of switching from locoregional to systemic therapies.

A comprehensive overview of systemic agents for advanced HCC (both approved and in development) is provided below.

\section{Systemic Therapy for Advanced HCC: First-Line Options}

\section{Sorafenib}

Sorafenib is an oral, small-molecule multiple tyrosine kinase inhibitor (TKI) that inhibits Raf-1, B-Raf, vascular endothelial growth factor receptor (VEGFR)1-3, and platelet-derived 


\section{Liver
Cancer}

\begin{tabular}{|c|c|}
\hline \multicolumn{2}{|c|}{-446} \\
\hline DOI: 10.1159/000499765 & $\begin{array}{l}\text { (c) } 2019 \text { S. Karger AG, Basel } \\
\text { www.karger.com/lic }\end{array}$ \\
\hline
\end{tabular}

Rimassa et al.: Systemic Treatment Options in HCC

growth factor receptor (PDGFR) $\beta$ - kinases involved in angiogenesis and tumor proliferation pathways implicated in the molecular pathogenesis of HCC [8].

In the phase III SHARP (Sorafenib Hepatocellular Carcinoma Assessment Randomized Protocol) trial $(n=602)$, sorafenib improved the overall survival (OS) versus placebo (median 10.7 vs. 7.9 months, respectively; hazard ratio [HR] $=0.69 ; 95 \%$ confidence interval [CI]) 0.55-0.87; $p<0.001$ ) in patients with advanced HCC (Child-Pugh A) who had not received prior systemic therapy [11]. The median time to radiologic progression (TTP) was significantly longer with sorafenib (5.5 vs. 2.8 months with placebo; HR $=0.58 ; 95 \%$ CI $0.45-0.74 ; p<0.001$ ). The disease control rate (DCR) was significantly higher with sorafenib (43 vs. $32 \%$ with placebo; $p=0.002$ ), although response rates (as per Response Evaluation Criteria in Solid Tumors [RECIST] v1.0) were low (sorafenib, 2\%; placebo, 1\%). Treatment-related adverse events (TRAE) with sorafenib occurred in $80 \%$ of the patients (vs. $52 \%$ with placebo); the most frequent grade $\geq 3$ TRAE ( $\geq 5 \%$ of the patients; sorafenib vs. placebo) were diarrhea ( 8 vs. $2 \%$ ) and hand-foot skin reaction (HFSR; 8 vs. $<1 \%$ ). TRAE led to discontinuation in 11 and $5 \%$ of sorafenib and placebo recipients, respectively. Based on these data, sorafenib was approved in 2007 for the treatment of patients with advanced HCC.

In the Sorafenib Asia-Pacific phase III trial that evaluated sorafenib versus placebo in advanced HCC $(n=226)$, HRs for OS (HR $=0.68 ; 95 \%$ CI $0.50-0.93 ; p=0.014)$ were similar to those in the SHARP trial, although the median OS was lower in both treatment arms (6.5 vs. 4.2 months) [12]. The large observational GIDEON (Global Investigation of Therapeutic Decisions in Hepatocellular Carcinoma and of its Treatment with Sorafenib) study of sorafenib in real-world clinical practice affirmed the safety and efficacy of sorafenib in a broader patient population, including the Child-Pugh B subpopulation; no significant additional toxicity was noted in this subgroup [13]. The use of sorafenib after failure of locoregional therapies is increasingly common; $57 \%$ of the patients in the GIDEON study had received prior surgical ablative therapy or TACE [14]. In a large prospective global observational study, patients who received sorafenib in this setting had a significantly longer OS than treatment-naïve patients (10.5 vs. 6.6 months; $p<0.001$ ) and were more likely to receive further anticancer treatment after sorafenib (31 vs. $9 \% ; p<0.001$ ) [14].

Post hoc analyses from the SHARP trial indicated that sorafenib was safe and effective irrespectively of the disease etiology, aspartate transaminase (AST)/alanine transaminase, $\alpha$-fetoprotein (AFP) and bilirubin levels, the baseline tumor burden, PS, tumor stage, and prior therapy $[15,16]$. Other pooled analyses showed that the magnitude of the OS benefit with sorafenib was greater in patients with HCV and lower neutrophil-to-lymphocyte ratios and patients whose disease was confined to the liver (without extrahepatic spread/macrovascular invasion) [17]. Data from a meta-analysis of 3 phase III trials also suggested that, unlike other targeted therapies, sorafenib is associated with improved OS in patients who are HCV positive and HBV negative; however, differential effects of sorafenib therapy, particularly etiologic patient differences, need to be assessed [18].

In a prospective analysis of sorafenib-treated patients $(n=147)$, early dermatologic adverse events (AE) (e.g., HFSR) occurring within 60 days of treatment initiation were shown to be associated with better survival outcomes [19]. The findings of a large observational study in nearly 5,000 patients suggest that AE (and the likelihood of treatment discontinuation) might be reduced by initiating sorafenib treatment at lower-than-recommended doses without significantly reducing the OS benefits [20].

Limitations of Sorafenib Treatment: Resistance

Despite the significant survival benefit seen with sorafenib in advanced HCC, this benefit is not sustained. Many patients who are treated with sorafenib experience disease progression 


\section{Liver

Rimassa et al.: Systemic Treatment Options in HCC

or discontinue the therapy because of $\mathrm{AE}[11,12,21]$. In an Italian observational study, $32 \%$ of the patients permanently discontinued sorafenib due to AE [21].

The prognosis is poor for patients who discontinue sorafenib therapy. In a study of 260 patients with advanced HCC, the median OS following sorafenib discontinuation was 4.1 months [22]. Liver dysfunction, PS, progression of target lesions, and the presence of new extrahepatic lesions was significantly correlated with postsorafenib survival [22, 23].

In addition, primary and acquired resistance to sorafenib severely limits OS benefits; in the SHARP trial approximately one quarter of the patients had primary resistance (with no initial response) to sorafenib [11]. The mechanisms of primary sorafenib resistance remain unclear but may include: overexpression and activation of epidermal growth factor receptor impacting downstream Ras/Raf/MEK/ERK pathways [24]; upregulation of stress-inducible protein Sestrin2, which activates Akt and AMPK signaling [25]; and a high expression of copine-III (CPNE3), possibly via the epithelial-mesenchymal transition process [26]. Mechanisms suggested to contribute to acquired sorafenib resistance include: abnormal activation of PI3K/Akt and JAK-STAT pathways; activation of hypoxia-inducible, alternative angiogenic, and fibroblast growth factor (FGF) signaling pathways; mesenchymal-epithelial transition receptor (c-MET) overexpression; genome instability; and epigenetic regulation $[27,28]$. In particular, c-MET overexpression has been shown to be a significant adverse prognostic factor in HCC patients undergoing surgical resection [29, 30].

Second- or later-line treatments that would potentially circumvent sorafenib resistance should be considered, particularly for patients with a good PS and reasonable liver function who may derive substantial benefits from such treatments.

\section{Lenvatinib}

Following the approval of sorafenib in 2007, clinical trials for first-line agents were unable to show improvement in outcomes versus sorafenib (online suppl. Table 1; for all online suppl. material, see www.karger.com/doi/10.1159/000499765) until 2017-2018, when lenvatinib demonstrated noninferiority versus sorafenib.

Lenvatinib is an oral, small-molecule TKI that targets VEGFR1-3, FGF receptor (FGFR)1-4, $\operatorname{PDGFR} \alpha / \beta$, KIT, and RET. Lenvatinib is distinguished from sorafenib in that it can target FGF signaling pathways in HCC [8].

Based on promising phase II results [31], the phase III noninferiority REFLECT trial of lenvatinib versus sorafenib was conducted in 954 patients (lenvatinib, $n=478$; sorafenib, $n=$ 476) with untreated advanced HCC (Child-Pugh A, excluding those with $\geq 50 \%$ liver occupation or bile duct/main portal vein invasion) [32]. REFLECT achieved its primary endpoint of noninferiority for OS; the median OS was 13.6 versus 12.3 months (HR $=0.92 ; 95 \%$ CI $0.79-1.06$ ) for lenvatinib versus sorafenib, respectively [32]. Patients treated with lenvatinib had a longer progression-free survival (PFS; 7.3 vs. 3.6 months; HR $=0.65$; 95\% CI 0.56-0.77; $p<0.0001$ ), TTP (7.4 vs. 3.7 months; $\mathrm{HR}=0.61 ; 95 \% \mathrm{CI} 0.51-0.72 ; p<0.0001$ ) and a higher objective response rate (ORR; 18.8 vs. $6.5 \% ; p<0.0001$ ) as per RECIST v1.1 compared to sorafenib [32].

Lenvatinib demonstrated similar rates of severe toxicity and delayed health-related quality-of-life (QoL) declines (in some domains) compared to sorafenib. The overall incidence of treatment-emergent AE (TEAE) was $99 \%$ in each arm. The most frequent grade $\geq 3 \mathrm{TEAE}$ ( $\geq 10 \%$ of the patients) with lenvatinib versus sorafenib were hypertension ( $23 \mathrm{vs.} 14 \%$ ) and HFSR (3 vs. 11\%). Overall, 9 versus $7 \%$ of the patients in the lenvatinib arm versus the sorafenib arm, respectively, discontinued treatment due to TRAE [32]. In 2018, lenvatinib was approved in Japan, the USA, and the European Union (EU) for the first-line treatment of unresectable HCC.

In an observational Japanese study of lenvatinib-treated patients with unresectable HCC, therapeutic responses and $\mathrm{AE}$ profiles were similar irrespectively of whether or not the 


\section{Liver Cancer}

\begin{tabular}{l|l}
\hline Liver Cancer 2019;8:427-446 \\
\hline DOI: 10.1159/000499765 & $\begin{array}{l}\text { @ 2019 S. Karger AG, Basel } \\
\text { www.karger.com/lic }\end{array}$ \\
\hline
\end{tabular}

Rimassa et al.: Systemic Treatment Options in HCC

patients had previously received another TKI [33]. The safety of lenvatinib in real-world clinical practice and combination regimens with immune checkpoint inhibitors (ICI) is being explored further in multiple ongoing trials (Tables 1, 2).

\section{Second-Line Systemic Therapy for Advanced HCC}

Second- and later-line treatments are needed for patients who fail or are intolerant to first-line treatment. From 2007 to 2016, most clinical trials for second-line agents were unable to show improvement in outcomes among sorafenib-treated patients (online suppl. Table 1).

However, rapid developments were noted beginning in 2017. Immune checkpoint inhibition is an area of expanding clinical development, with scores of trials of immunotherapeutic agents (as monotherapy or combinations) ongoing or recently completed in different settings and disease stages, including the first line [34]. Multiple phase II/III trials reported positive results, and as a result of this approvals were obtained for regorafenib (USA, EU, and Japan), nivolumab (USA; accelerated approval), cabozantinib (EU), and pembrolizumab (USA; accelerated approval) for patients with disease progression on or intolerance to sorafenib.

These recent clinical advances, discussed below, have major implications in how oncologists/hepatologists treat patients with advanced HCC.

\section{Recently Approved Agents}

Regorafenib

Regorafenib is an oral, small-molecule multikinase inhibitor of VEGFR1-3, TIE2, PDGFR $\beta$, FGFR, RET, KIT, RAF kinase, and MAPK - kinases involved in angiogenesis, oncogenesis, metastasis, and tumor immunity $[8,35]$. Regorafenib had a distinct molecular target profile from sorafenib in preclinical studies and was expected to have more profound antiangiogenic activity due to the combined inhibition of vascular endothelial growth factor (VEGF) and TIE pathways [35].

The phase III RESORCE (Regorafenib after Sorafenib in Patients with Hepatocellular Carcinoma) trial evaluated regorafenib versus placebo in 573 patients (regorafenib, $n=379$; placebo, $n=194$ ) with HCC (Child-Pugh A) whose disease had progressed on sorafenib [36]. RESORCE met its primary endpoint; regorafenib improved the median OS over placebo (10.6 vs. 7.8 months, respectively; $\mathrm{HR}=0.63 ; 95 \% \mathrm{CI} 0.50-0.79 ; p<0.0001$ ). In the regorafenib arm versus the placebo arm, respectively, as per RECIST v1.1, the overall response rate was 7 versus 3\%, the DCR was 66 versus 35\% ( $p<0.0001)$, and the median PFS was 3.4 versus 1.5 months (HR $=0.43 ; 95 \%$ CI 0.35-0.52; $p<0.0001)$. TEAE occurred in 100 and 93\% of regorafenib and placebo recipients, respectively. The most common grade 3 or higher TEAE (in $\geq 10 \%$ of patients) with regorafenib versus placebo were HFSR (13 vs. 1\%), hypertension (15 vs. 5\%), increased AST (11 vs. 11\%), and increased blood bilirubin (10 vs. 11\%). Overall, 10 versus $4 \%$ of the patients discontinued treatment due to TRAE in the regorafenib arm versus the placebo arm, respectively. Based on these data, regorafenib was approved in the USA, the EU, and Japan for patients with HCC previously treated with sorafenib [36].

Of note, patients in the RESORCE trial were required to tolerate $\geq 400 \mathrm{mg}$ sorafenib/day for at least 20 days in the last 28 days of treatment. Therefore, it is uncertain whether regorafenib will be well tolerated or effective among sorafenib-intolerant patients and whether these patients may show a similar pattern of intolerance towards regorafenib.

Post hoc analyses of the RESORCE trial showed that regorafenib conferred a clinical benefit regardless of the last sorafenib dose or TTP with prior sorafenib and the safety profile of regorafenib did not vary when analyzed based on the last sorafenib dose [37]. Like sorafenib [19], skin toxicity (specifically HFSR) with regorafenib was associated with improved OS [38]. 


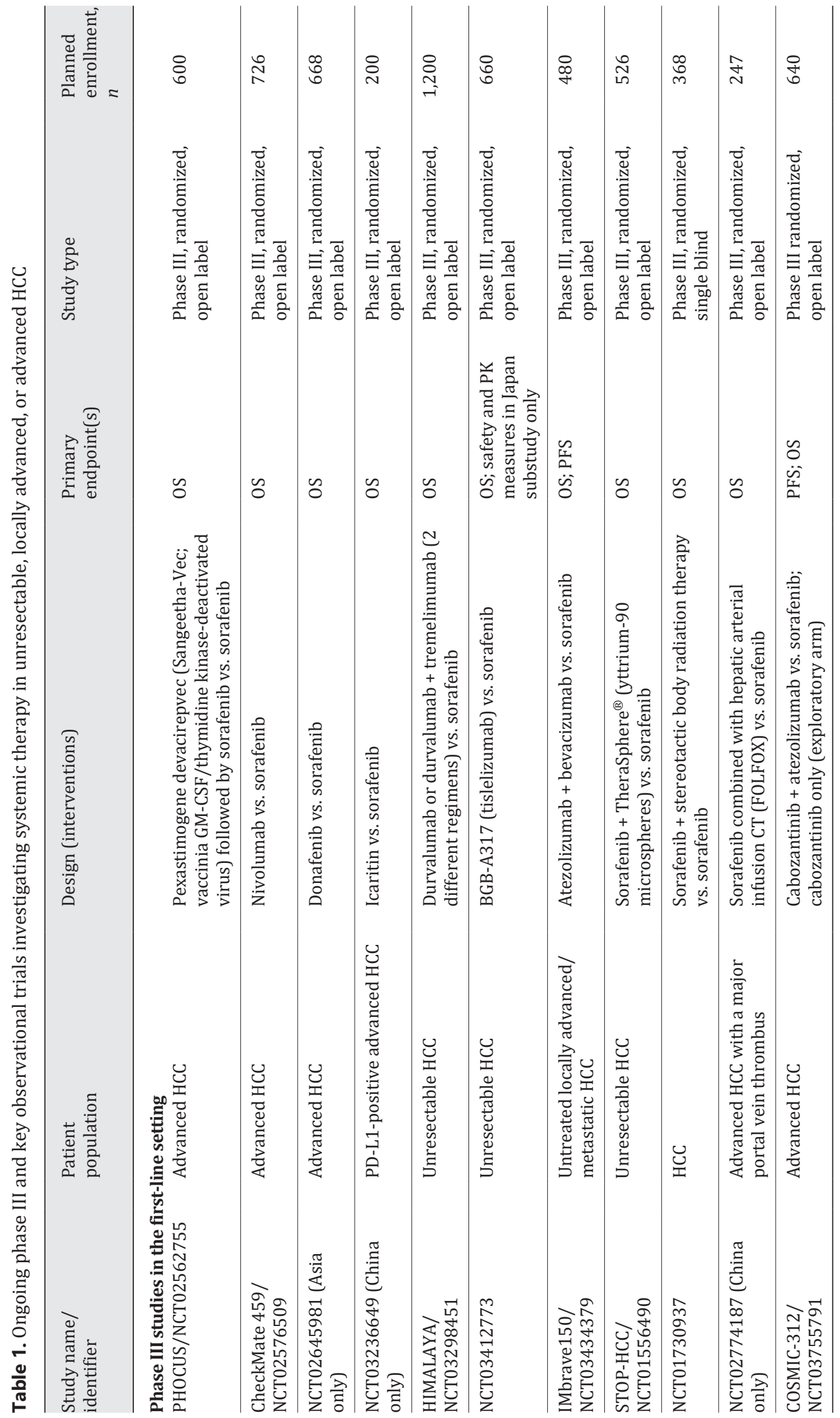




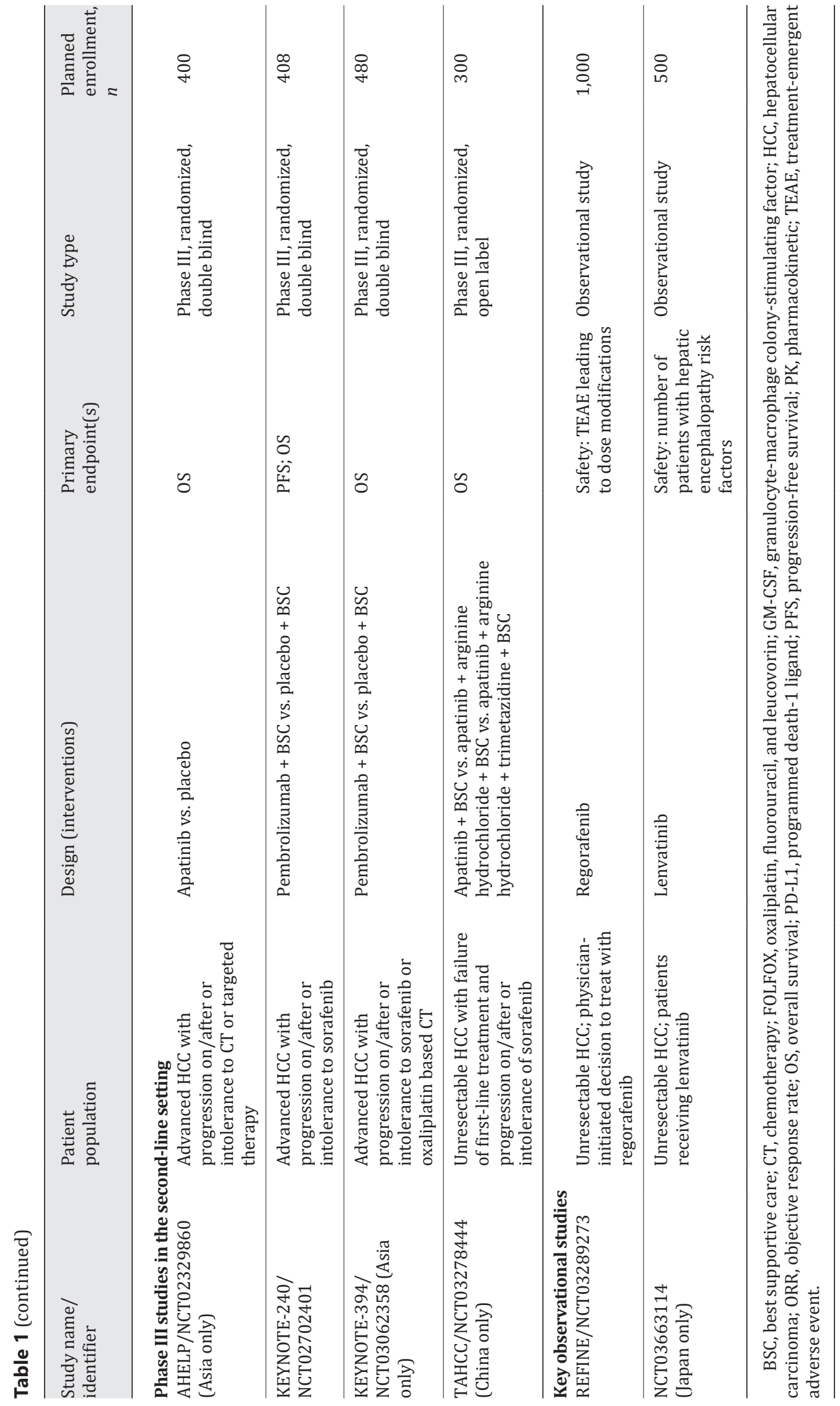




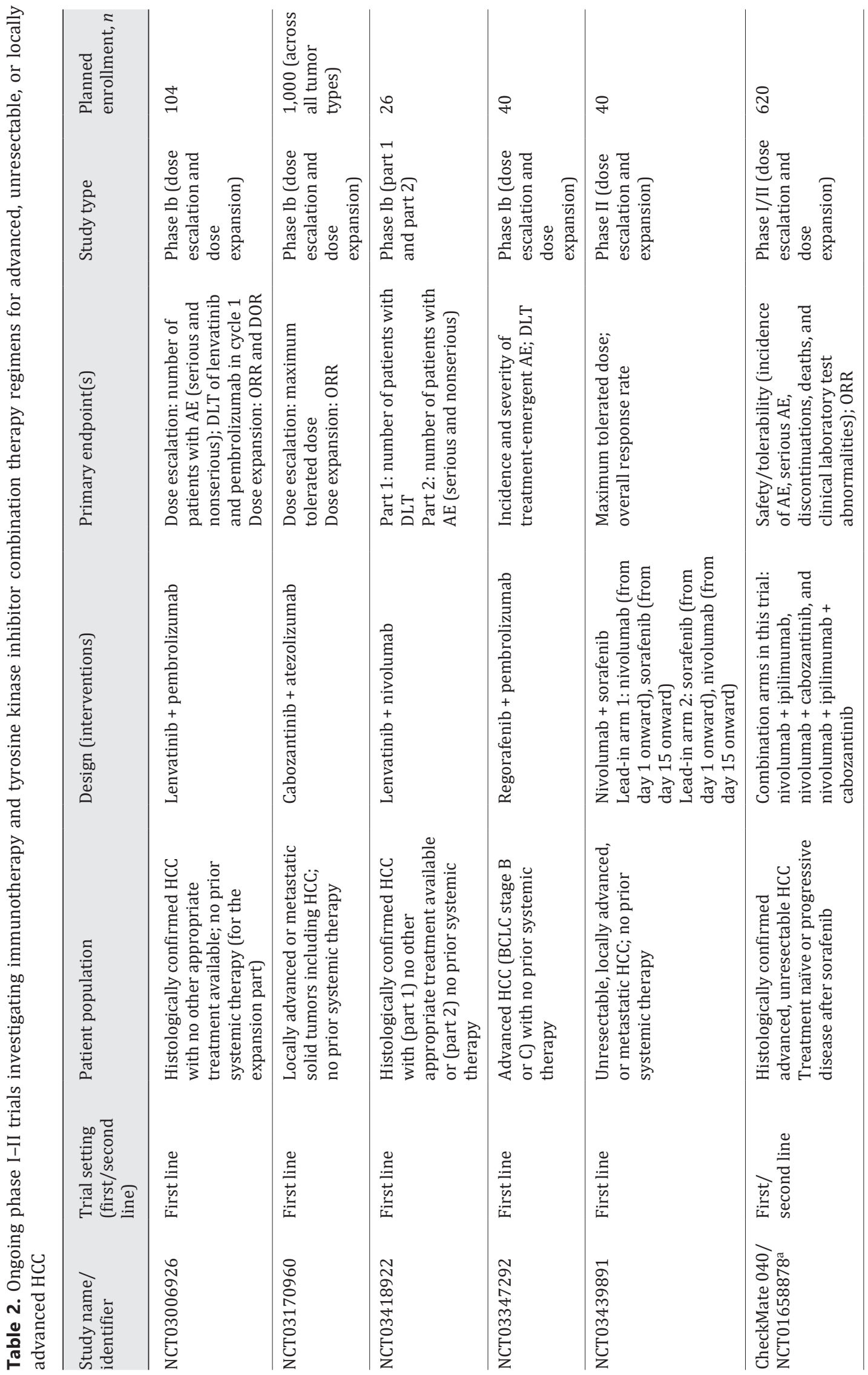



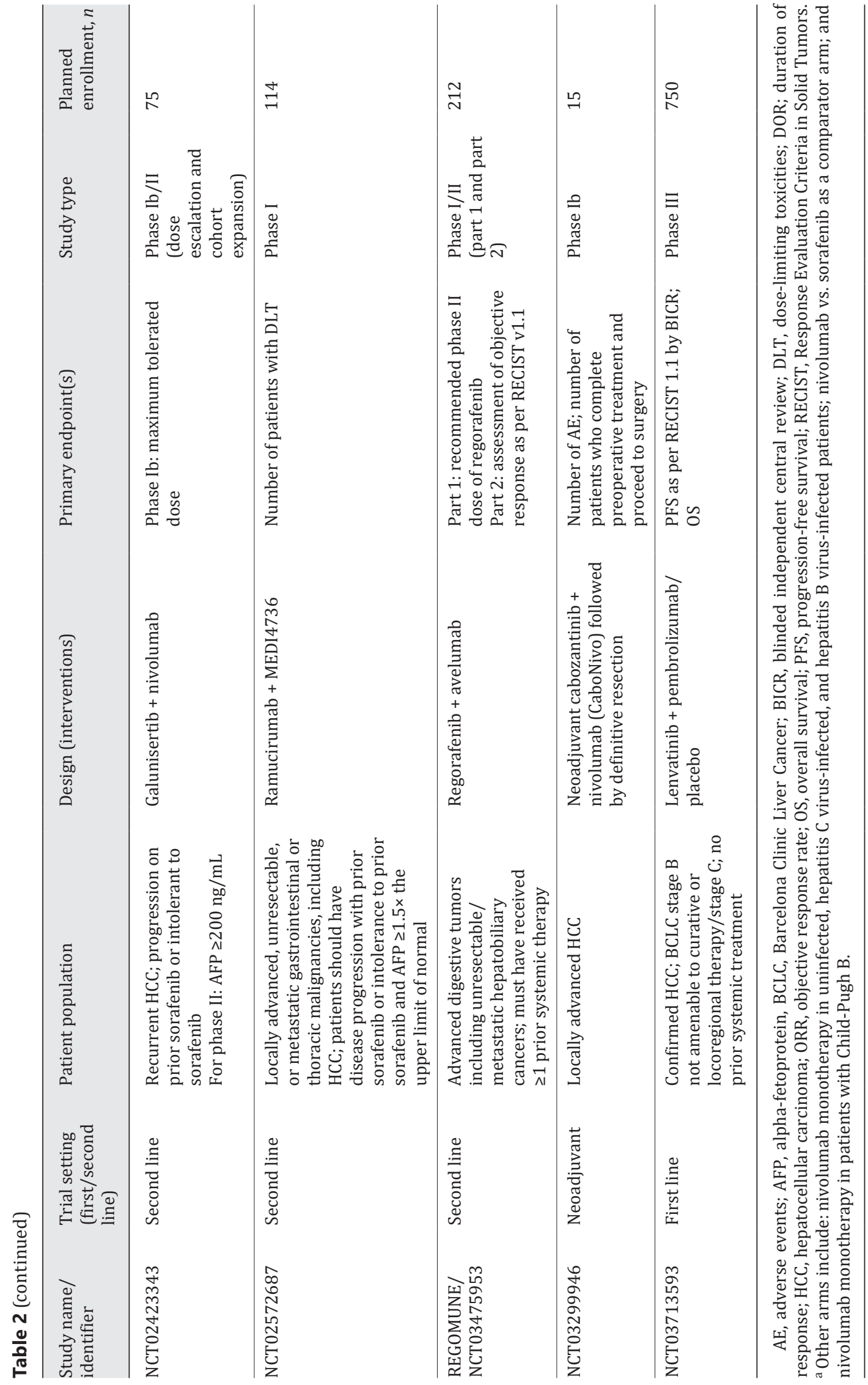


\section{Liver

Rimassa et al.: Systemic Treatment Options in HCC

Regorafenib showed benefits in patients with HCC regardless of baseline AFP or c-MET expression levels [39].

REFINE (Regorafenib Observational Study in Hepatocellular Carcinoma; NCT03289273), an ongoing observational study, is evaluating regorafenib in real-world clinical practice (Table 1). Regorafenib is also being evaluated in combination with ICI in multiple trials (Table 2).

Nivolumab

The immunobiology of HCC renders this tumor type a good candidate for ICI. Immune tolerance in HCC tumors includes myeloid suppression, upregulation of the programmed death (PD)-1 pathway, and induction or recruitment of T-regulatory cells. Patients whose tumors had better T-cell infiltration had a longer survival and a lower risk of recurrence [40, 41].

Nivolumab, an anti-PD-1 inhibitor antibody, helps to restore the antitumor immune response and has demonstrated a survival benefit across several tumor types [41]. The phase I/ II CheckMate 040 study assessed nivolumab in patients with histologically confirmed advanced HCC (Child-Pugh A to B7) [42] in the following cohorts: prior sorafenib treatment (yes/no), HCV, and HBV. The primary endpoints were safety and tolerability in the phase I dose escalation phase ( $n=48$ ) and ORR (as per RECIST v1.1) in the dose expansion phase ( $n=214)$.

Nivolumab demonstrated a manageable safety profile. In the dose escalation phase, 83 and $25 \%$ of the patients reported any-grade and grade $3 / 4$ TRAE, respectively, and the maximum tolerated dose was not reached. In the dose expansion phase, $19 \%$ of the patients experienced grade $3 / 4$ TRAE and $11 \%$ of the patients discontinued the therapy due to AE; the median PFS was 4.0 months (95\% CI 2.9-5.4) [42]. With a longer follow-up (median $=12.9$ months) the investigator-assessed ORR was $23 \%$, the median duration of response (DOR) was not reached, and the 12 -month OS rate was $73 \%$ among sorafenib-naive patients $(n=80)$ across both the dose expansion and the dose escalation cohorts. Among sorafenib-experienced patients in the dose escalation $(n=37)$ and dose expansion cohorts $(n=145)$, respectively, investigator-assessed ORR were 16 and 19\%; the median DOR were 17 and 12 months, and the 12-month OS rates were 58 and $60 \%$, with a longer follow up. Responses occurred regardless of the etiology or PD-ligand 1 (PD-L1) expression [43].

Based on the ORR and the durability of the response, nivolumab was granted accelerated approval in the USA for patients with previously treated HCC; approvals in EU/Japan are pending confirmatory data.

Ongoing trials are evaluating nivolumab as monotherapy in first-line HCC (phase III CheckMate 459 [NCT02576509]: nivolumab vs. sorafenib) and in combination with other agents in first- and second-line settings (Tables 1,2).

Cabozantinib

Cabozantinib, an oral, small-molecule TKI, is active against VEGFR2, c-MET, and AXL, as well as RET, KIT, and FLT3. These kinases are implicated in tumor angiogenesis, survival, and metastasis and in pathways involved in resistance to VEGFR inhibitors, including sorafenib [8].

In a phase II trial, cabozantinib demonstrated clinical activity including objective responses, disease stabilization, and reductions in AFP in patients with advanced HCC and $\leq 1$ prior systemic anticancer regimen [44]. The phase III CELESTIAL trial evaluated cabozantinib versus placebo in 707 patients (cabozantinib, $n=470$; placebo, $n=237$ ) with advanced HCC (Child-Pugh A) who had received up to 2 prior systemic regimens (including prior sorafenib) and had disease progression [45]. CELESTIAL met its primary endpoint; cabozantinib significantly improved the OS versus placebo (median 10.2 vs. 8.0 months, respectively; $H R=0.76$; $95 \%$ CI $0.63-0.92 ; p=0.005$ ). The OS benefit was more pronounced in patients who had received sorafenib as the only prior systemic therapy (11.3 vs. 7.2 months, cabozantinib vs. 


\section{Liver
Cancer}

\begin{tabular}{|c|c|}
\hline \multicolumn{2}{|c|}{-446} \\
\hline DOI: 10.1159/000499765 & $\begin{array}{l}\text { (c) } 2019 \text { S. Karger AG, Basel } \\
\text { www.karger.com/lic }\end{array}$ \\
\hline
\end{tabular}

Rimassa et al.: Systemic Treatment Options in HCC

placebo; HR $=0.70 ; 95 \%$ CI $0.55-0.88)$. Cabozantinib also significantly improved the PFS (median 5.2 vs. 1.9 months with placebo; HR $=0.44 ; 95 \%$ CI $0.36-0.52 ; p<0.001$ ) and the ORR as per RECIST v1.1 ( 4 vs. $0.4 \%$ with placebo; $p=0.009$ ). A consistent PFS benefit was observed across all predefined subgroups.

In the cabozantinib versus placebo groups, respectively, TEAE were reported in 99 versus $92 \%$ of the patients, and TRAE resulted in discontinuation in 16 versus $3 \%$ of the patients. The most common grade $3 / 4$ TEAE with cabozantinib versus placebo were HFSR (17 vs. 0\%), hypertension (16 vs. $2 \%$ ), increased AST (12 vs. $7 \%$ ), fatigue (10 vs. $4 \%$ ), and diarrhea (10 vs. $2 \%$ ). Based on these results, in November 2018, the European Medicines Agency (EMA) approved cabozantinib for the treatment of advanced HCC in adults previously treated with sorafenib.

Several trials are currently exploring the combination of cabozantinib with ICI in advanced HCC, including nivolumab and atezolizumab (Tables 1, 2).

Pembrolizumab

Pembrolizumab, a PD-1 inhibitor antibody [41], was evaluated in patients with advanced HCC $(n=104)$, with intolerance to or disease progression with sorafenib in the phase II KEYNOTE-224 trial [46]. Pembrolizumab resulted in durable objective responses (ORR as per RECIST v1.1: 17\%; the median DOR was not reached [range 3.1-14.6+ months]), a median PFS of 4.9 months, and a median OS of 12.9 months [46]. Overall, TRAE were reported in $73 \%$ of the patients; the most common grade $3 / 4$ TRAE, occurring in $\geq 5 \%$ of the patients, was increased AST (7\%). Overall, $5 \%$ of the patients discontinued the therapy due to AE. Based on these data, the US Food and Drug Administration (FDA) granted accelerated approval to pembrolizumab for patients with HCC previously treated with sorafenib.

Pembrolizumab is currently being evaluated in second-line advanced HCC in the ongoing phase III KEYNOTE-240 trial (NCT02702401; Table 1) and in combination with TKI in phase I/II trials (Table 2).

\section{Agents with Positive Phase III Trial Results}

Ramucirumab

Ramucirumab, a humanized monoclonal antibody that selectively inhibits VEGFR2, showed activity in HCC in early phase I trials [47]. A preplanned analysis of the phase III REACH trial, which evaluated ramucirumab versus placebo in previously treated advanced HCC, indicated a significant survival benefit with ramucirumab in a subgroup of patients with elevated AFP levels [48].

This formed the basis for the phase III biomarker-driven REACH-2 trial that evaluated ramucirumab versus placebo in patients (ramucirumab, $n=197$; placebo, $n=95$ ) with advanced HCC (with disease progression on or intolerance to sorafenib) and elevated baseline AFP levels $(\geq 400 \mathrm{ng} / \mathrm{mL})$ [49]. REACH-2 met its primary endpoint; treatment with ramucirumab significantly improved the OS (median 8.5 vs. 7.3 months with placebo; HR $=0.710$; 95\% CI 0.531-0.949; $p=0.0199$ ). Ramucirumab also improved the PFS as per RECIST v1.1 (median 2.8 vs. 1.6 months with placebo; HR $=0.452 ; 95 \%$ CI $0.339-0.603 ; p<0.0001$ ) and DCR (as per RECIST v1.1; 59.9 vs. $38.9 \%$ with placebo; $p=0.0006$ ).

A pooled efficacy and safety analysis of patients from the REACH- 2 trial and patients with AFP $\geq 400 \mathrm{ng} / \mathrm{mL}$ from the REACH trial (ramucirumab, $n=316$; placebo, $n=226$ ) confirmed these findings [50]. Ramucirumab treatment resulted in significant improvements in OS (median 8.1 vs. 5.0 months with placebo; $\mathrm{HR}=0.694 ; 95 \%$ CI $0.571-0.842 ; p=0.0002$ ), $\mathrm{PFS}$ (median 2.8 vs. 1.5 months with placebo; HR $=0.572 ; 95 \%$ CI $0.472-0.694 ; p<0.0001$ ), and DCR (56.3 vs. $37.2 \%$ with placebo; $p<0.0001)$. Ramucirumab demonstrated a manageable safety profile. In the ramucirumab arm versus the placebo arm, respectively, the most common grade 3 or higher $\mathrm{AE}$ were hypertension (12 vs. $4 \%$ ) and hyponatremia ( 5 vs. $2 \%$ ); overall, 9.5 versus $3.6 \%$ of the 


\section{Liver

Rimassa et al.: Systemic Treatment Options in HCC

patients discontinued the treatment due to TRAE. Ramucirumab also showed declines in diseaserelated symptoms relative to placebo in the pooled patient population [51], making it one of the first second-line agents to demonstrate both OS and QoL benefits.

REACH-2 was the first phase III biomarker-driven trial in advanced HCC to achieve its primary endpoint; the METIV-HCC trial of tivantinib versus placebo in a c-MET-high biomarker-selected population in second-line HCC had negative results [52].

Immunotherapy and TKI Combination Regimens: Rationale and Promise

While the activity of TKI remains relatively modest in HCC $[11,36]$, expectations are high for ICI in advanced HCC, considering promising preliminary data $[42,46]$ and positive results in other solid malignancies [53,54]. As the HCC microenvironment has several active immune tolerance pathways, there is evidence to suggest that combining therapeutic modalities may offer a greater benefit than monotherapy $[40,41]$.

Combined inhibition of the PD-1 and cytotoxic T-lymphocyte-associated antigen-4 (CTLA-4) redundant immune checkpoint pathways has shown efficacy across tumor types [41]. This combination is being evaluated in the ongoing phase III HIMALAYA trial (NCT03298451; durvalumab [PD-L1 inhibitor] plus tremelimumab [CTLA-4 inhibitor] in first-line unresectable HCC) and the CheckMate 040 trial (nivolumab plus ipilimumab in second-line advanced HCC) (Tables 1, 2).

Preclinical studies indicated that bevacizumab, the anti-VEGF antibody, may further enhance PD-1/PD-L1 efficacy by reversing VEGF-mediated immunosuppression and promoting tumor T-cell infiltration [55]. This rationale was supported by results from phase III trials of atezolizumab, the PD-L1 inhibitor antibody, plus bevacizumab in renal cell carcinoma [56] and nonsquamous non-small cell lung cancer [57]. However, the primary endpoint of PFS was not significantly increased in patients treated with bevacizumab plus atezolizumab versus bevacizumab in the phase II MODUL study in patients with metastatic colorectal cancer [58]; however, colorectal cancer is not a good paradigm due to its resistance to immunotherapy [59]. Nevertheless, initial results from a phase Ib study (NCT02715531) evaluating bevacizumab plus atezolizumab in unresectable metastatic HCC indicated promising response rates (ORR $=32 \% ; n=73$ ) and tolerability for this combination [60] and supported the ongoing phase III IMbrave150 trial (NCT03434379; atezolizumab plus bevacizumab vs. sorafenib in first-line advanced HCC; Table 1).

Multikinase inhibitors such as regorafenib may promote dendritic cell maturation and T-cell priming, activation, and differentiation into long-lived memory $\mathrm{T}$ cells by increasing tumor antigenicity and tumor immunogenicity [61]. Neoangiogenesis inhibition may result in increased adhesion molecule expression on tumor endothelial cells and increased intratumoral immune cell trafficking and infiltration [40]. Regorafenib also reduces inhibitory tumor-associated macrophage cell levels, thereby reducing their immunosuppressive effects by inhibiting the colony-stimulating factor 1 receptor, which appears to play a role in the tumor association of macrophages [61, 62]. Similarly, inhibition of angiopoietin/TIE2 signaling, which is involved in tumor angiogenesis, may also modulate inhibitory tumor-associated macrophage cells in the tumor microenvironment [62, 63]. The addition of PD-1 inhibitors may serve to consolidate clinical responses into long-lasting clinical remissions by blocking the coinhibitory receptors PD-1/PD-L1/PD-L2 and precluding tumor immune escape [41]. Taken together, these data support the scientific rationale for the combination of regorafenib and pembrolizumab in an ongoing phase Ib/II trial in the first-line setting (NCT03347292; Table 2). Similarly, in a phase Ib trial, the combination of lenvatinib and pembrolizumab demonstrated promising response rates in patients with advanced HCC [64], and this combination is being evaluated further in the first line (NCT03713593; Table 2). 


\section{Liver
Cancer}

\begin{tabular}{|c|c|}
\hline \multicolumn{2}{|c|}{ Liver Cancer 2019;8:427-446 } \\
\hline DOI: 10.1159/000499765 & $\begin{array}{l}\text { (c) } 2019 \text { S. Karger AG, Basel } \\
\text { www.karger.com/lic }\end{array}$ \\
\hline
\end{tabular}

Rimassa et al.: Systemic Treatment Options in HCC

Cabozantinib was shown to modulate the expression of tumor cell markers associated with immune recognition in murine colon carcinoma cell lines [65]. Cabozantinib altered the composition of the peripheral immune environment by increasing $\mathrm{CD} 8^{+} \mathrm{T}$ cells, reducing T-regulatory cells and myeloid-derived suppressor cells, and increasing the sensitivity of murine tumor cells to T-cell-mediated killing. These results suggest immune-modulating capabilities for cabozantinib and support its clinical combination with cancer immunotherapy. The combination of cabozantinib and immunotherapy is being investigated in multiple ongoing trials in HCC (Tables 1,2), including the phase III COSMIC-312 trial (NCT03755791).

To improve on the $20 \%$ ORR initially seen with PD-L1 inhibitors, nivolumab is being evaluated in first- or later-line settings in combination with sorafenib, mogamulizumab (CCR4 inhibitor), lirilumab (killer cell immunoglobulin-like receptor inhibitor), galunisertib (transforming growth factor- $\beta$ inhibitor) and pexa-vec (an antitumor multipeptide vaccine), and avelumab is being evaluated in combination with the VEGFR inhibitor axitinib in phase I/II trials in patients with advanced HCC [34].

\section{Challenges in the Treatment of Advanced HCC}

\section{Assessment of Clinical Efficacy}

OS remains the most important measure of clinical efficacy in HCC and it is the primary endpoint for most phase III trials. In an analysis of postprogression survival of patients who were treated with or discontinued sorafenib, OS had a greater correlation with patterns of HCC progression than tumor burden changes $[22,23]$. TTP did not always correlate with OS in sorafenib trials [66]. Similarly, recent analyses from the regorafenib RESORCE trial showed a poor correlation between ORR/TTP and OS [67]. These analyses suggest that ORR and TTP are not reliable surrogate endpoints for OS in advanced HCC, although they are often used for assessing the therapeutic benefit in clinical practice. However, in the REFLECT study of lenvatinib versus sorafenib in patients with HCC, objective response assessed by modified RECIST (mRECIST) criteria irrespectively of treatment was an independent predictor of OS [68].

There is some controversy regarding the appropriate criteria for the assessment of response. RECIST v1.1 are most widely used across trials for the assessment of tumor size changes; however, these criteria do not take into consideration devascularization and necrosis [69] and can sometimes be unreliable for assessing the tumor burden. mRECIST criteria were developed specifically for HCC, as these criteria take into account tumor devascularization/ necrosis following ablation or TACE [70]. Although mRECIST is well established as efficacy marker for local ablations or TACE, the superiority of mRECIST over RECIST v1.1 is not established for systemic therapies. Immune-related RECIST criteria are being considered for tumors treated with ICI, as the response patterns with these agents are unique [71]; however, even for these agents, OS appears to be the best way to gauge benefit.

\section{Patient Selection: Biomarkers and Patient Stratification}

The success of clinical trials necessitates careful patient selection and stratification in their study designs. For instance, in second-line trials, it is important to stratify patients according to the reason for sorafenib withdrawal/failure [22,23]. The absence/presence of extrahepatic spread and macrovascular invasion may help to inform the pattern of progression and predict the OS in these patients [23].

Due to the clinical and molecular heterogeneity of HCC, the use of biomarkers to select patients who may benefit from treatment is far from established [8], although recent developments indicate promise for biomarker-driven therapies. 
Rimassa et al.: Systemic Treatment Options in HCC

AFP is the most widely used biomarker in HCC and it is typically regarded as a predictor of recurrence and survival, particularly in advanced HCC [8]. The biomarker-driven phase III REACH-2 trial demonstrated significantly improved outcomes with ramucirumab versus placebo in patients with elevated baseline AFP levels [49]. c-MET did not show promise as a biomarker in second-line advanced HCC. Tivantinib showed OS and PFS benefits in a subgroup of patients with high c-MET-expressing HCC in a phase II trial, but in the phase III trial tivantinib did not improve OS or PFS versus placebo in patients with a high c-MET expression [52, 72].

PD-L1 is emerging as a possible biomarker for the response to PD-1 inhibitors in HCC; however, the utility of PD-L1 as a biomarker varies across tumor types and depending on the PD-1 inhibitor used. In the CheckMate 040 trial, objective responses occurred with nivolumab regardless of tumor PD-L1 expression [42], while in KEYNOTE-224 the response to pembrolizumab was associated with PD-L1 expression in a subset of patients $[42,46]$. Results from the ongoing phase III trials of PD- 1 and CTLA- 4 agents will help to clarify the role/need for additional biomarker testing when immunotherapeutic agents are employed in the treatment of HCC.

\section{Expert Opinion: Changing Treatment Algorithms for Patients with Advanced HCC}

\section{Multidisciplinary Treatment Approach}

HCC is a complex disease that requires a multidisciplinary treatment approach at all BCLC stages, involving the expertise of liver and transplant surgeons, interventional radiologists skilled both in standard and innovative techniques (endovascular therapies /percutaneous ablations), hepatologists and medical oncologists with expertise in TKI and immunotherapy, and radiation oncologists experienced in external beam conformal or stereotactic radiotherapy. These healthcare professionals must work together to develop effective treatment strategies, taking into consideration not only the patient's current HCC lesions but also possible outcomes, including relapse, progression, and treatment failures.

\section{Choice and Sequence of Treatment for Advanced HCC}

Patients

Systemic therapy should be selected for patients whose liver functions are preserved and who have good PS (ECOG PS 0 or 1). This highlights the need to limit prior locoregional therapies in these patients to avoid irreversible deterioration of liver function or general health status. For instance, it is recommended that patients switch to systemic therapy if no response is observed after TACE. Patients with HCC should refrain from alcohol consumption and they should undergo treatment for HBV infection, diabetes, and hypertension, which are risk factors that affect disease progression and treatment.

Choice of First-Line Treatment

With lenvatinib receiving approval, there are now 2 available options for first-line treatment, i.e., sorafenib and lenvatinib. In the REFLECT trial, the safety profiles of both drugs were very similar [32], and QoL analysis showed minor improvements with lenvatinib versus sorafenib. Although lenvantinib was superior to sorafenib for the secondary endpoints (PFS, TTP, and ORR), the optimum choice of treatment in clinical practice remains unclear, particularly since there are no available biomarkers to help inform the choice of first-line treatment at this time. Biomarker analyses performed in lenvatinib- and sorafenib-treated patients were hypothesis generating and need to be confirmed [37, 73]. Additional biomarker analyses and real-world data on safety are needed to help clarify these choices in clinical practice. 


\section{Liver
Cancer}

\begin{tabular}{|c|c|}
\hline \multicolumn{2}{|c|}{-446} \\
\hline DOI: 10.1159/000499765 & $\begin{array}{l}\text { (c) } 2019 \text { S. Karger AG, Basel } \\
\text { www.karger.com/lic }\end{array}$ \\
\hline
\end{tabular}

Rimassa et al.: Systemic Treatment Options in HCC

Immunotherapy (alone or in combination with TKI) may also be a likely first-line option in the near future, pending results from the CheckMate 459, HIMALAYA, and IMbrave trials (Tables 1, 2).

Choice and Sequence of Second-Line Treatment

With the approval of regorafenib (in the USA and the EU), nivolumab (USA), cabozantinib (EU), and pembrolizumab (USA), there are now multiple second-line treatment options available for patients with advanced HCC who have failed first-line treatment.

The choice of second-line treatment and the sequence of first-, second-, and later-line treatments for advanced HCC remain complicated, primarily due to the lack of evidence. Second-line agents have mostly been explored in patients with disease progression on or intolerance to sorafenib. Tolerance profiles of the different first-line agents (sorafenib and lenvatinib) are important considerations in the choice of second-line treatments.

Thus far, the most convincing treatment sequence is sorafenib, followed by regorafenib. Among patients who were treated with regorafenib following sorafenib, the median OS was 26.0 months from the beginning of sorafenib treatment (vs. 19.2 months for placebo following sorafenib; the prior treatment time on sorafenib was similar for both groups) [37]. The efficacy of regorafenib is uncertain in sorafenib-intolerant patients, as discussed earlier [36].

Cabozantinib may be a good choice for patients irrespectively of their tolerance to sorafenib. Biomarker analyses from the phase III CELESTIAL trial are pending and will provide insight into whether patients with c-MET overexpression (associated with sorafenib resistance) $[24,25]$ may potentially be good candidates for cabozantinib (which also targets the c-MET and AXL pathways). Although patients who were both tolerant and intolerant to sorafenib were enrolled into the CELESTIAL trial, specific data for each of these patient subgroups were not collected. Prospective clinical trials are needed to validate this strategy and to evaluate c-MET as a biomarker for response to cabozantinib. At this time, cabozantinib is also the only agent to have been evaluated in a third-line setting, although other agents may likely be effective in this setting. Both cabozantinib and regorafenib need to be carefully considered in patients prone to arterial hypertension $[36,45]$.

While both cabozantinib and regorafenib were effective in patients with advanced HCC and a high AFP expression, ramucirumab is the only drug that has been specifically evaluated and has demonstrated efficacy in HCC patients with a high AFP expression. In the absence of comparative data between these agents, ramucirumab may be considered the best choice for patients with AFP $\geq 400 \mathrm{ng} / \mathrm{mL}$ in the event of approval [49]. While ramucirumab was well tolerated in the REACH-2 trial [49], patients receiving ramucirumab should be carefully monitored for hypertension, similar to cabozantinib and regorafenib. In addition, as ramucirumab is administered as an intravenous infusion, unlike other oral TKI, patient adherence is a consideration for choosing this agent.

At this time, there is uncertainty regarding the choice of second-line treatments following lenvatinib, as all drugs approved in the second-line setting were evaluated following sorafenib. Safety profiles and preliminary biomarker analyses were not informative for possible second-line treatment choices following lenvatinib [32]. A post hoc analysis of the treatment sequence after lenvatinib or sorafenib in the phase III REFLECT trial showed that treatment with any subsequent anticancer medication after first-line lenvatinib resulted in a median OS of 21 months versus 17 months after first-line sorafenib [74].The ongoing phase II trial (NCT03433703), which is currently investigating the efficacy of second-line treatments (as determined by the physician) after progression/unacceptable toxicity on lenvatinib, and other ongoing trials should provide insight into second-line treatments following lenvatinib. 
Immune Checkpoint Inhibitors

The results with ICI in HCC have been largely promising, and immuno-oncology agents (nivolumab and pembrolizumab) are important second-line treatment options after sorafenib, although phase III data for both drugs are pending. THE Results expected from CheckMate 459 and combination immunotherapy trials may lead to future immuno-oncology approvals in the first-line setting.

While durable responses are noted for patients who respond to ICI, only a small percentage of patients typically respond to treatment. The lack of definitive biomarkers impairs our ability to identify patients who may derive the greatest benefit from immunotherapy.

Additional innovative immunotherapeutic strategies in the second-line setting (targeting CTLA-4, TIM-3, LAG-3, and NK cell enhancers) may be explored for treating patients who are unresponsive to ICI in the first-line setting.

\section{Conclusions}

The last 2 years have seen important positive developments in the treatment landscape of advanced HCC, with the approval of several new agents. With additional imminent approvals based on recent and ongoing phase III trials, patients may have access to more treatment choices in the future. However, decisions including the choice, timing, and sequence of treatment are complex and will need to be made carefully to ensure the optimal patient benefit. It is critical that healthcare professionals be educated on rapidly evolving changes and that they be aware of the complexity of treatment choices and sequencing. With new and promising combination regimens, it is anticipated that the treatment of advanced HCC may undergo further dramatic changes over the next 2 or 3 years. Multidisciplinary expertise is of the utmost importance to set up an optimal treatment strategy for each patient.

\section{Acknowledgement}

Editorial/medical writing support was provided by Vasupradha Vethantham, $\mathrm{PhD}$, of inScience Communications, Springer Healthcare (New York, NY, USA).

\section{Statement of Ethics}

The authors have no ethical conflicts to disclose.

\section{Disclosure Statement}

Lorenza Rimassa declares advisory/consulting for Arqule, Bayer, Celgene, Eli Lilly, Eisai, Exelixis, Incyte, Ipsen, and Sirtex Medical and speaker activities for AstraZeneca, AbbVie, Gilead, and Roche. Tiziana Pressiani has no conflicts of interests to declare. Philippe Merle declares advisory/consulting for Bayer, Bristol-Myers Squibb, Ely Lilly, Exelixis, IPSEN, Genosciences, Merck, Nanobiotix, Onxeo, and Roche.

\section{Funding Sources}

Funding for medical writing support was provided by Exelixis, Inc.

\section{Author Contributions}

All of the authors performed the research, writing, and review of all of the drafts of this paper and approved the final version. 
Rimassa et al.: Systemic Treatment Options in HCC

\section{References}

1 Forner A, Reig M, Bruix J. Hepatocellular carcinoma. Lancet. 2018 Mar;391(10127):1301-14.

2 Yau T, Tang VY, Yao TJ, Fan ST, Lo CM, Poon RT. Development of Hong Kong Liver Cancer staging system with treatment stratification for patients with hepatocellular carcinoma. Gastroenterology. 2014;146: 1691-1700.e1693.

3 Piscaglia F, Ogasawara S. Patient selection for transarterial chemoembolization in hepatocellular carcinoma: importance of benefit/risk assessment. Liver Cancer. 2018 Mar;7(1):104-19.

4 Omata M, Cheng AL, Kokudo N, Kudo M, Lee JM, Jia J, et al. Asia-Pacific clinical practice guidelines on the management of hepatocellular carcinoma: a 2017 update. Hepatol Int. 2017 Jul;11(4):317-70.

5 Kudo M, Moriguchi M, Numata K, Hidaka H, Tanaka H, Ikeda M, et al. S-1 versus placebo in patients with sorafenib-refractory advanced hepatocellular carcinoma (S-CUBE): a randomised, double-blind, multicentre, phase 3 trial. Lancet Gastroenterol Hepatol. 2017 Jun;2(6):407-17.

6 Wörns MA, Galle PR. Hepatocellular carcinoma in 2017: two large steps forward, one small step back. Nat Rev Gastroenterol Hepatol. 2018 Feb;15(2):74-6.

7 Peck-Radosavljevic M, Kudo M, RaouL JL, Lee HC, Decaens T, Heo J, et al. Outcomes of patients (pts) with hepatocellular carcinoma (HCC) treated with transarterial chemoembolization (TACE): Global OPTIMIS final analysis. J Clin Oncol. 2018;36(15 suppl):4018.

8 Llovet JM, Zucman-Rossi J, Pikarsky E, Sangro B, Schwartz M, Sherman M, et al. Hepatocellular carcinoma. Nat Rev Dis Primers. 2016 Apr;2:16018.

9 Arizumi T, Ueshima K, Minami T, Kono M, Chishina H, Takita M, et al. Effectiveness of sorafenib in patients with transcatheter arterial chemoembolization (TACE) refractory and intermediate-stage hepatocellular carcinoma. Liver Cancer. 2015 Dec;4(4):253-62.

10 Ogasawara S, Chiba T, Ooka Y, Kanogawa N, Motoyama T, Suzuki E, et al. Efficacy of sorafenib in intermediate-stage hepatocellular carcinoma patients refractory to transarterial chemoembolization. Oncology. 2014;87(6):330-41.

11 Llovet JM, Ricci S, Mazzaferro V, Hilgard P, Gane E, Blanc JF, et al.; SHARP Investigators Study Group. Sorafenib in advanced hepatocellular carcinoma. N Engl J Med. 2008 Jul;359(4):378-90.

12 Cheng AL, Kang YK, Chen Z, Tsao CJ, Qin S, Kim JS, et al. Efficacy and safety of sorafenib in patients in the Asia-Pacific region with advanced hepatocellular carcinoma: a phase III randomised, double-blind, placebocontrolled trial. Lancet Oncol. 2009 Jan;10(1):25-34.

13 Marrero JA, Kudo M, Venook AP, Ye SL, Bronowicki JP, Chen XP, et al. Observational registry of sorafenib use in clinical practice across Child-Pugh subgroups: the GIDEON study. J Hepatol. 2016 Dec;65(6):1140-7.

14 Yen C, Sharma R, Rimassa L, Arizumi T, Bettinger D, Choo HY, et al. Treatment stage migration maximizes survival outcomes in patients with hepatocellular carcinoma treated with sorafenib: an observational study. Liver Cancer. 2017 Nov;6(4):313-24.

15 Bruix J, Raoul JL, Sherman M, Mazzaferro V, Bolondi L, Craxi A, et al. Efficacy and safety of sorafenib in patients with advanced hepatocellular carcinoma: subanalyses of a phase III trial. J Hepatol. 2012 Oct; 57(4): 821-9.

16 Raoul JL, Bruix J, Greten TF, Sherman M, Mazzaferro V, Hilgard P, et al. Relationship between baseline hepatic status and outcome, and effect of sorafenib on liver function: SHARP trial subanalyses. J Hepatol. 2012 May;56(5):1080-8.

17 Bruix J, Cheng AL, Meinhardt G, Nakajima K, De Sanctis Y, Llovet J. Prognostic factors and predictors of sorafenib benefit in patients with hepatocellular carcinoma: analysis of two phase III studies. J Hepatol. 2017 Nov;67(5):999-1008.

18 Jackson R, Psarelli EE, Berhane S, Khan H, Johnson P. Impact of viral status on survival in patients receiving sorafenib for advanced hepatocellular cancer: a meta-analysis of randomized phase III trials. J Clin Oncol. 2017 Feb;35(6):622-8.

19 Reig M, Torres F, Rodriguez-Lope C, Forner A, LLarch N, Rimola J, et al. Early dermatologic adverse events predict better outcome in HCC patients treated with sorafenib. J Hepatol. 2014 Aug;61(2):318-24.

20 Reiss KA, Yu S, Mamtani R, Mehta R, D'Addeo K, Wileyto EP, et al. Starting dose of sorafenib for the treatment of hepatocellular carcinoma: a retrospective, multi-institutional study. J Clin Oncol. 2017 Nov;35(31): 3575-81.

21 Iavarone M, Cabibbo G, Piscaglia F, Zavaglia C, Grieco A, Villa E, et al.; SOFIA (SOraFenib Italian Assessment) study group. Field-practice study of sorafenib therapy for hepatocellular carcinoma: a prospective multicenter study in Italy. Hepatology. 2011 Dec;54(6):2055-63.

22 Iavarone M, Cabibbo G, Biolato M, Della Corte C, Maida M, Barbara M, et al. Predictors of survival in patients with advanced hepatocellular carcinoma who permanently discontinued sorafenib. Hepatology. 2015 Sep; 62(3):784-91.

23 Reig M, Rimola J, Torres F, Darnell A, Rodriguez-Lope C, Forner A, et al. Postprogression survival of patients with advanced hepatocellular carcinoma: rationale for second-line trial design. Hepatology. 2013 Dec; 58(6):2023-31.

24 Ezzoukhry Z, Louandre C, Trécherel E, Godin C, Chauffert B, Dupont S, et al. EGFR activation is a potential determinant of primary resistance of hepatocellular carcinoma cells to sorafenib. Int J Cancer. 2012 Dec; 131(12):2961-9. 
25 Dai J, Huang Q, Niu K, Wang B, Li Y, Dai C, et al. Sestrin 2 confers primary resistance to sorafenib by simultaneously activating AKT and AMPK in hepatocellular carcinoma. Cancer Med. 2018 Nov;7(11):5691-703.

26 Chen Z, Jiang Z, Zhang W, He B. Silencing the expression of copine-III enhances the sensitivity of hepatocellular carcinoma cells to the molecular targeted agent sorafenib. Cancer Manag Res. 2018 Aug;10:3057-67.

27 Zhu YJ, Zheng B, Wang HY, Chen L. New knowledge of the mechanisms of sorafenib resistance in liver cancer. Acta Pharmacol Sin. 2017 May;38(5):614-22.

28 Hu G, Zhang Y, Ouyang K, Xie F, Fang H, Yang X, et al. In vivo acquired sorafenib-resistant patient-derived tumor model displays alternative angiogenic pathways, multi-drug resistance and chromosome instability. Oncol Lett. 2018 Sep;16(3):3439-46.

$29 \mathrm{Kim} \mathrm{JH}, \mathrm{Kim}$ HS, Kim BJ, Jang HJ, Lee J. Prognostic value of c-Met overexpression in hepatocellular carcinoma: a meta-analysis and review. Oncotarget. 2017 Aug;8(52):90351-7.

30 Rimassa L, Abbadessa G, Personeni N, Porta C, Borbath I, Daniele B, et al. Tumor and circulating biomarkers in patients with second-line hepatocellular carcinoma from the randomized phase II study with tivantinib. Oncotarget. 2016 Nov; 7(45):72622-33.

31 Ikeda K, Kudo M, Kawazoe S, Osaki Y, Ikeda M, Okusaka T, et al. Phase 2 study of lenvatinib in patients with advanced hepatocellular carcinoma. J Gastroenterol. 2017 Apr;52(4):512-9.

32 Kudo M, Finn RS, Qin S, Han KH, Ikeda K, Piscaglia F, et al. Lenvatinib versus sorafenib in first-line treatment of patients with unresectable hepatocellular carcinoma: a randomised phase 3 non-inferiority trial. Lancet. 2018 Mar;391(10126):1163-73.

33 Hiraoka A, Kumada T, Kariyama K, Takaguchi K, Atsukawa M, Itobayashi E, et al.; Real-life Practice Experts for HCC (RELPEC) Study Group, HCC 48 Group (hepatocellular carcinoma experts from 48 clinics in Japan). Clinical features of lenvatinib for unresectable hepatocellular carcinoma in real-world conditions: multicenter analysis. Cancer Med. 2019 Jan;8(1):137-46.

34 Flynn MJ, Sayed AA, Sharma R, Siddique A, Pinato DJ. Challenges and opportunities in the clinical development of immune checkpoint inhibitors for hepatocellular carcinoma. Hepatology. 2018 Nov. https://doi. org/10.1002/hep.30337.

35 Wilhelm SM, Dumas J, Adnane L, Lynch M, Carter CA, Schütz G, et al. Regorafenib (BAY 73-4506): a new oral multikinase inhibitor of angiogenic, stromal and oncogenic receptor tyrosine kinases with potent preclinical antitumor activity. Int J Cancer. 2011 Jul;129(1):245-55.

36 Bruix J, Qin S, Merle P, Granito A, Huang YH, Bodoky G, et al.; RESORCE Investigators. Regorafenib for patients with hepatocellular carcinoma who progressed on sorafenib treatment (RESORCE): a randomised, double-blind, placebo-controlled, phase 3 trial. Lancet. 2017 Jan;389(10064):56-66.

37 Finn RS, Merle P, Granito A, Huang YH, Bodoky G, Pracht M, et al. Outcomes of sequential treatment with sorafenib followed by regorafenib for HCC: additional analyses from the phase III RESORCE trial. J Hepatol. 2018 Aug;69(2):353-8.

38 Bruix J, Merle P, Granito A, Huang YH, Bodoky G, Yokosuka O, et al.; RESORCE Investigators. Hand-foot skin reaction (HFSR) and overall survival (OS) in the phase 3 RESORCE trial of regorafenib for treatment of hepatocellular carcinoma (HCC) progressing on sorafenib. J Clin Oncol. 2018;36:412.

39 Teufel M, Köchert K, Meinhardt G, Bruix J. Efficacy of regorafenib (REG) in patients with hepatocellular carcinoma (HCC) in the phase III RESORCE trial according to alpha-fetoprotein (AFP) and c-Met levels as predictors of poor prognosis. J Clin Oncol. 2017;35:4078.

40 Pardee AD, Butterfield LH. Immunotherapy of hepatocellular carcinoma: unique challenges and clinical opportunities. OncoImmunology. 2012 Jan;1(1):48-55.

41 Kudo M. Immune checkpoint inhibition in hepatocellular carcinoma: basics and ongoing clinical trials. Oncology. 2017;92:50-62.

42 El-Khoueiry AB, Sangro B, Yau T, Crocenzi TS, Kudo M, Hsu C, et al. Nivolumab in patients with advanced hepatocellular carcinoma (CheckMate 040): an open-label, non-comparative, phase $1 / 2$ dose escalation and expansion trial. Lancet. 2017 Jun;389(10088):2492-502.

43 Crocenzi TS, El-Khoueiry AB, Yau TC, Melero I, Sangro B, Kudo M, et al. Nivolumab (nivo) in sorafenib (sor)naive and -experienced pts with advanced hepatocellular carcinoma (HCC): CheckMate 040 study. J Clin Oncol. 2017;35:4013.

44 Kelley RK, Verslype C, Cohn AL, Yang TS, Su WC, Burris H, et al. Cabozantinib in hepatocellular carcinoma: results of a phase 2 placebo-controlled randomized discontinuation study. Ann Oncol. 2017 Mar;28(3):528-34.

45 Abou-Alfa GK, Meyer T, Cheng AL, El-Khoueiry AB, Rimassa L, Ryoo BY, et al. Cabozantinib in patients with advanced and progressing hepatocellular carcinoma. N Engl J Med. 2018 Jul;379(1):54-63.

46 Zhu AX, Finn RS, Edeline J, Cattan S, Ogasawara S, Palmer D, et al.; KEYNOTE-224 investigators. Pembrolizumab in patients with advanced hepatocellular carcinoma previously treated with sorafenib (KEYNOTE224): a non-randomised, open-label phase 2 trial. Lancet Oncol. 2018 Jul;19(7):940-52.

47 Spratlin JL, Cohen RB, Eadens M, Gore L, Camidge DR, Diab S, et al. Phase I pharmacologic and biologic study of ramucirumab (IMC-1121B), a fully human immunoglobulin G1 monoclonal antibody targeting the vascular endothelial growth factor receptor-2. J Clin Oncol. 2010 Feb;28(5):780-7.

48 Zhu AX, Park JO, Ryoo BY, Yen CJ, Poon R, Pastorelli D, et al.; REACH Trial Investigators. Ramucirumab versus placebo as second-line treatment in patients with advanced hepatocellular carcinoma following first-line therapy with sorafenib (REACH): a randomised, double-blind, multicentre, phase 3 trial. Lancet Oncol. 2015 Jul;16(7):859-70. 
Rimassa et al.: Systemic Treatment Options in HCC

49 Zhu AX, Kang YK, Yen CJ, Finn RS, Galle PR, Llovet JM, et al.; REACH-2 study investigators. Ramucirumab after sorafenib in patients with advanced hepatocellular carcinoma and increased $\alpha$-fetoprotein concentrations (REACH-2): a randomised, double-blind, placebo-controlled, phase 3 trial. Lancet Oncol. 2019 Feb; 20(2):282-96.

50 Zhu A, Finn R, Galle P, Llovet J, Blanc JF, Okusaka T, et al. Ramucirumab as second-line treatment in patients with advanced hepatocellular carcinoma (HCC) and elevated alpha-fetoprotein (AFP) following first-line sorafenib: pooled efficacy and safety across two global randomized phase 3 studies (REACH- 2 and REACH). Ann Oncol. 2018;29:208.

51 Zhu AX, Finn RS, Galle PR, Llovet JM, Blanc JF, Okusaka T, Chau I, Cella D, Girvan A, Gable J, Bowman L, Hsu Y, Abada PB, Kudo M. Ramucirumab as second-line treatment in patients with advanced hepatocellular carcinoma (HCC) and elevated alpha-fetoprotein (AFP) following first-line sorafenib: patient reported outcome results across two phase III studies (REACH-2 and REACH). Ann Oncol. 2018;29:282.

52 Rimassa L, Assenat E, Peck-Radosavljevic M, Pracht M, Zagonel V, Mathurin P, et al. Tivantinib for secondline treatment of MET-high, advanced hepatocellular carcinoma (METIV-HCC): a final analysis of a phase 3, randomised, placebo-controlled study. Lancet Oncol. 2018 May;19(5):682-93.

53 Assi HI, Kamphorst AO, Moukalled NM, Ramalingam SS. Immune checkpoint inhibitors in advanced nonsmall cell lung cancer. Cancer. 2018 Jan;124(2):248-61.

54 Furue M, Ito T, Wada N, Wada M, Kadono T, Uchi H. Melanoma and immune checkpoint inhibitors. Curr Oncol Rep. 2018 Mar;20(3):29.

55 Wallin JJ, Bendell JC, Funke R, Sznol M, Korski K, Jones S, et al. Atezolizumab in combination with bevacizumab enhances antigen-specific T-cell migration in metastatic renal cell carcinoma. Nat Commun. 2016 Aug; $7(1): 12624$.

56 Motzer RJ, Powles T, Atkins MB, Escudier B, McDermott DF, Suarez C, et al. IMmotion151: a randomized phase III study of atezolizumab plus bevacizumab vs sunitinib in untreated metastatic renal cell carcinoma (mRCC). J Clin Oncol. 2018;36:578.

57 Reck M, Socinski MA, Cappuzzo F, Orlandi F, Stroyakovskii D, Nogami N, Rodríguez-Abreu D, Moro-Sibilot D, Thomas CA, Barlesi F, Finley G, Kelsch C, Lee A, Coleman S, Shen Y, Kowanetz M, Lopez-Chavez A, Sandler A, Jotte R. Primary PFS and safety analyses of a randomized phase III study of carboplatin + paclitaxel +/bevacizumab, with or without atezolizumab in $1 \mathrm{~L}$ non-squamous metastatic nsclc (IMPOWER150). Ann Oncol. 2017;28:760.

58 Grothey A, Tabernero J, Arnold D, De Gramont A, Ducreux MP, O'Dwyer PJ, Van Cutsem E, Bosanac I, Srock S, Mancao C, Gilberg F, Winter J. Fluoropyrimidine (FP) + bevacizumab (BEV) + atezolizumab vs FP/BEV in BRAFwt metastatic colorectal cancer (mCRC): findings from Cohort 2 of MODUL - a multicentre, randomized trial of biomarker-driven maintenance treatment following first-line induction therapy. Ann Oncol. 2018; 29:LBA19.

59 Le DT, Hubbard-Lucey VM, Morse MA, Heery CR, Dwyer A, Marsilje TH, et al. A blueprint to advance colorectal cancer immunotherapies. Cancer Immunol Res. 2017 Nov;5(11):942-9.

60 Pishvaian MJ, Lee MS, Ryoo BY, Stein S, Lee KH, Verret W, et al. Updated safety and clinical activity results from a phase Ib study of atezolizumab + bevacizumab in hepatocellular carcinoma (HCC): Paper presented at European Society for Medical Oncology 2018 Congress; October 19-23. Munich, Germany, 2018

61 Tsai AK, Khan AY, Worgo CE, Wang LL, Liang Y, Davila E. A multikinase and DNA-PK inhibitor combination immunomodulates melanomas, suppresses tumor progression, and enhances immunotherapies. Cancer Immunol Res. 2017 Sep;5(9):790-803.

62 Noy R, Pollard JW. Tumor-associated macrophages: from mechanisms to therapy. Immunity. 2014 Jul; 41(1):49-61.

63 Hughes PE, Caenepeel S, Wu LC. Targeted therapy and checkpoint immunotherapy combinations for the treatment of cancer. Trends Immunol. 2016 Jul;37(7):462-76.

64 Ikeda M, Sung MW, Kudo M, Kobayashi M, Baron AD, Finn RS, et al. A phase $1 \mathrm{~b}$ trial of lenvatinib (LEN) plus pembrolizumab (PEM) in patients (pts) with unresectable hepatocellular carcinoma (uHCC). J Clin Oncol. 2018;36:4076.

65 Kwilas AR, Ardiani A, Donahue RN, Aftab DT, Hodge JW. Dual effects of a targeted small-molecule inhibitor (cabozantinib) on immune-mediated killing of tumor cells and immune tumor microenvironment permissiveness when combined with a cancer vaccine. J Transl Med. 2014 Nov;12(1):294.

66 Huang L, Sanctis YD, Shan M, Bruix J, Llovet J, Cheng AL, et al. Weak correlation of overall survival and time to progression in advanced hepatocellular carcinoma. J Clin Oncol. 2017;35:233.

67 Bruix J, Huang LP, Nakajima K, Han GH, Meinhardt G, Merle P. Time to progression (TTP) and response rate (RR) are not reliable surrogate endpoints for overall survival (OS) in hepatocellular carcinoma (HCC): an analysis from the phase 3 RESORCE trial. Hepatology. 2018;68:169a.

68 Kudo M, Finn RS, Qin S, Ikeda K, Cheng AL, Piscaglia F, et al. Analysis of survival and objective response (OR) in patients with hepatocellular carcinoma in a phase III study of lenvatinib (REFLECT). J Clin Oncol 2019; 37:186.

69 Bruix J, Reig M, Sangro B. Assessment of treatment efficacy in hepatocellular carcinoma: response rate, delay in progression or none of them. J Hepatol. 2017 Jun;66(6):1114-7.

70 Lencioni R, Llovet JM. Modified RECIST (mRECIST) assessment for hepatocellular carcinoma. Semin Liver Dis. 2010 Feb;30(1):52-60. 
71 Hodi FS, Hwu WJ, Kefford R, Weber JS, Daud A, Hamid O, et al. Evaluation of immune-related response criteria and RECIST v1.1 in patients with advanced melanoma treated with pembrolizumab. J Clin Oncol. 2016 May;34(13):1510-7.

72 Santoro A, Rimassa L, Borbath I, Daniele B, Salvagni S, Van Laethem JL, et al. Tivantinib for second-line treatment of advanced hepatocellular carcinoma: a randomised, placebo-controlled phase 2 study. Lancet Oncol. 2013 Jan;14(1):55-63.

73 Finn RS, Kudo M, Cheng AL, Wyrwicz L, Ngan R, Blanc JF, Baron AD, Vogel A, Ikeda M, Piscaglia F, Han KH, Qin S, Minoshima Y, Kanekiyo M, Ren M, Dairiki R, Tamai T, Dutcus CE, Funahashi Y, Evans TRJ. Final analysis of serum biomarkers in patients (pts) from the phase III study of lenvatinib (LEN) vs sorafenib (SOR) in unresectable hepatocellular carcinoma (uHCC) (REFLECT). Ann Oncol. 2018;29:269.

74 Alsina A, Kudo M, Vogel A, Cheng AL, Tak WY, Ryoo BY, et al. Subsequent anticancer medication following first-line lenvatinib: A posthoc responder analysis from the phase 3 REFLECT study in unresectable hepatocellular carcinoma. J Clin Oncol. 2019;37:371. 